\author{
Hanno Hoppe · Peter Vock • Harald Marcel Bonel • \\ Christoph Ozdoba $\cdot$ Jan Gralla
}

\title{
A novel multiple-trauma CT-scanning protocol using patient repositioning
}

Received: 28 February 2006 / Accepted: 17 March 2006 / Published online: 13 October 2006

(C) Am Soc Emergency Radiol 2006

\begin{abstract}
Emergency CT examination is considered to be a trade-off between a short scan time and the acceptance of artifacts. This study evaluates the influence of patient repositioning on artifacts and scan time. Eighty-three consecutive multiple-trauma patients were included in this prospective study. Patients were examined without repositioning (group 1, $n=39$ ) or with patient rotation to feetfirst with arms raised for scanning the chest and abdomen/ pelvis (group 2, $n=44$ ). The mean scan time was $21 \mathrm{~min}$ in group 1 and $25 \mathrm{~min}$ in group $2(P=0.01)$. The mean repositioning time in group 2 was 8 min. Significantly, more artifacts were observed in group 1 (with a repeated scan in $7 \%)$ than in group $2(P=0.0001)$. This novel multiple- trauma CT-scanning protocol with patient repositioning achieves a higher image quality with significantly fewer artifacts than without repositioning but increases scan time slightly.
\end{abstract}

Keywords Multidetector computed tomography · Multiple trauma $\cdot$ Emergency $\cdot$ CT protocol $\cdot$ Workflow

\section{Introduction}

At present, computed tomography (CT) plays a major role in diagnostic imaging of tertiary care emergency services [1-4]. Of all emergency conditions, the multiple-trauma patient has perhaps benefited most from the introduction of multi-row detector CT, due to shorter scan times and a high

\footnotetext{
H. Hoppe $\cdot$ P. Vock $\cdot$ H. M. Bonel $\cdot$ J. Gralla Department of Interventional and Diagnostic Radiology, University Hospital of Bern, Freiburgstrasse 4,

CH-3010 Bern, Switzerland

C. Ozdoba · J. Gralla $(\bowtie)$

Department of Interventional and Diagnostic Neuroradiology,

University Hospital of Bern,

Bern, Switzerland

e-mail: jan.gralla@insel.ch

Tel.: +41-31-6322655

Fax: +41-31-6324872
}

spatial resolution [5-8]. Many centers are, therefore, equipped with dedicated CT scanners to allow fast access for emergency patients, especially those with multiple trauma [9].

The choice of protocol for the CT examination of multiple-trauma patients entails a trade-off between a short scan time and the acceptance of artifacts. For multipletrauma patients, the total time spent in the CT suite through early diagnosis to initiation of treatment has a profound influence on the patient's outcome $[10,11]$. On the other hand, it is crucial for image quality that the optimal protocol is chosen, especially with regard to patient positioning [12].

During a whole body scan, repositioning of the patient to reduce artifacts caused by arms or installations may improve image quality, but it can also prolong the scan time [12].

In this study, a novel protocol with patient rotation from head-first and arms at the side for scanning the head and neck to feet-first with arms raised for scanning the chest, abdomen and pelvis [13] was compared to a head-first CT protocol without repositioning [12,14]. To our knowledge, this is the first study to prospectively compare these protocols measuring time intervals and assessing image quality.

\section{Materials and methods}

Patients

The study design accords with the guidelines of our institute's ethics committee and was performed according to regulations outlined in the revised Declaration of Helsinki of 1998. Multiple trauma was defined as two or more severe injuries in at least two areas of the body. Eighty-three consecutive multiple-trauma patients were prospectively assigned to two groups based on the time of their examination (Table 1): Group 1 consisted of patients who were scanned without repositioning $(n=39)$, group 2 of patients scanned with repositioning $(n=44)$ from the head- 
first position with arms at the side for scanning the head and neck to the feet-first position with raised arms for scanning the chest, abdomen, and pelvis. Some data from group 2 had already been discussed previously [13].

\section{CT examination}

Patients were scanned using a 16-row detector CT scanner (Sensation16, Siemens, Erlangen, Germany) installed in the emergency department. The scanner has a minimal rotation time of $0.5 \mathrm{~s}$ with a collimation between 0.75 and $1.5 \mathrm{~mm}$. This allowed rapid scanning of large body segments in short scanning times.

The scanning protocol for multiple-trauma patients including segmented scanning of the head/neck and body is summarized in Table 2. Head and neck scans were planned on a first scout and scanned without administration of intravenous contrast medium. Helical CT scans of the chest, pelvis, and abdomen were planned on a second scout. For evaluation of an abdominal arterial and portal venous phase within one scan, contrast agent was applied via multiple boluses. A total of $140 \mathrm{ml}$ of iopromide contrast medium containing $300 \mathrm{mg} / \mathrm{ml}$ iodine (Ultravist 300, Berlex Laboratories, Montville, NJ, USA) was powerinjected intravenously. In a first phase, $80 \mathrm{ml}$ of contrast medium was injected with a scan delay of $50 \mathrm{~s}$ and a flowrate of $3 \mathrm{ml} / \mathrm{s}$. In a second phase, $60 \mathrm{ml}$ of contrast medium was injected with a scan delay of $10 \mathrm{~s}$ and a flow-rate of $4 \mathrm{ml} / \mathrm{s}$. Except for head scans, tube current was automatically adapted using the scanner's "Care Dose" mode for minimizing radiation exposure.

Patients in group 1 were examined in head-first position with arms at the side and without repositioning. In the event major artifacts due to installations or arms did not allow conclusive evaluation, a repeat scan of the abdomen with raised arms was performed and an additional contrast bolus of $80 \mathrm{ml}$ applied.

Patients in group 2 were initially positioned in the scanner head-first with arms at the side for examination of the head and neck. For subsequent imaging of the body, they were rotated to a feet-first position and their arms raised to reduce artifacts caused by the arms themselves, or by electrodes or cables within the scan field. The emergency personnel were instructed in advance when and how to rotate the patient on the CT table. Before the examination, a plastic board was positioned below the vacuum cushion to permit patient repositioning. Fractures and/or dislocations of the arms and/or shoulders were clinically ruled out before the CT scan. If fractures and/or dislocations of the arms and/or shoulders were suspected, the patient's arms were not raised during the CT examination and the patient was assigned to group 1 so as not to engender harm to this patient who could have his injuries made worse by manipulating the upper extremities in repositioning and turning.

A board-certified radiologist supervised the CT examination. For image reading and reformatting a workstation (Volume Wizard, Somaris 5 VA, Siemens, Erlangen, Germany) was used. Images were viewed with regular soft tissue contrast window display settings (level $50 \mathrm{H}$, width $450 \mathrm{H}$ ), bone window settings (level 450, width 1,500 ), and lung window settings (level $-450 \mathrm{H}$, width $1,850 \mathrm{H}$ ). If necessary, the window settings were adjusted manually at the workstation.

\section{Definition of time intervals}

Time intervals were measured for study purposes by medical personnel not involved in patient treatment. The following time intervals were recorded:

(1) Scan time: from the start of first scout measurement until the radiologist's decision to conclude the actual CT examination. This time interval includes planning of the examination based on the scout images, patient repositioning, application of contrast agent, and the radiologist's decision process to conclude the examination.

(2) Repositioning time: a subdivision of the scan time in group 2, during which the patient was rotated from head-first to feet-first position.

\section{Artifacts}

Images were retrospectively graded by two radiologists as a consensus reading. Artifacts were graded semiquantitatively as follows: grade $0-$ no substantial

Table 1 Characteristics of patients without (group 1) and with (group 2) repositioning

\begin{tabular}{lllll}
\hline & & Total & Group 1 (no repositioning) & Group 2 (repositioning) \\
\hline $\begin{array}{l}\text { Number of patients } \\
\text { Sex }\end{array}$ & Male/female & 83 & 39 & 44 \\
& & $65 / 18$ & $29 / 10$ & $36 / 8$ \\
Age & & $P=0.44$ & 44.4 \\
& Mean & 46.4 & 48.5 & $16-83$ \\
& Range & $16-89$ & $18-89$ & 21.3 \\
& Standard deviation & 21.4 & 21.5 & $38-51$ \\
& $\mathrm{CI}^{\mathrm{a}}$ & $41.7-51$ & $41.5-55.5$ & $P=0.4(95 \% \mathrm{CI}=-13.5-5.3)$ \\
\hline
\end{tabular}

${ }^{\mathrm{a}} \mathrm{CI}=95 \%$ confidence interval 
Table 2 Scanning parameters for adult multiple-trauma patients

\begin{tabular}{|c|c|c|c|c|c|c|c|c|}
\hline Body region $^{a}$ & Scan mode & Collimation (mm) & Rotation time (s) & Scan time $(\mathrm{s})^{\mathrm{b}}$ & Pitch & $\begin{array}{l}\text { Table feed } \\
\mathrm{mm} / \text { rotation }\end{array}$ & Tube current/energy ${ }^{c}$ & $\begin{array}{l}\text { Contrast } \\
\text { application }\end{array}$ \\
\hline Head & Sequential & 1.5 & 1.5 & 30 & 1 & 6 & $120 \mathrm{kV} 270 \mathrm{mAs}$ & N/A \\
\hline Neck & Helical & 0.75 & 0.75 & 14 & 2 & 6 & $120 \mathrm{kV} 330 \mathrm{mAs}$ & \\
\hline Chest & Helical & 1.5 & 0.5 & 8 & 1.3 & 30 & $120 \mathrm{kV} 200 \mathrm{mAs}$ & $\begin{array}{l}80 \mathrm{ml} 3 \mathrm{ml} / \mathrm{s} \\
\text { delay } 50 \mathrm{~s}\end{array}$ \\
\hline Abdomen & Helical & 1.5 & 0.5 & 10 & 1.3 & 24 & & $\begin{array}{l}60 \mathrm{ml} 4 \mathrm{ml} / \mathrm{s} \\
\text { delay } 10 \mathrm{~s}\end{array}$ \\
\hline Pelvis & Helical & 1.5 & 0.5 & 5 & 1.3 & 24 & & \\
\hline
\end{tabular}

${ }^{\mathrm{a}}$ Two separate scouts were acquired for the head/neck and body scan

${ }^{b}$ Depending on length of region; here, an approximation for adult patients

"Care Dose" was applied for dose optimization except for head scan

artifacts, grade $1-$ moderate artifacts (not impairing a diagnostic evaluation of liver and spleen), and grade 2 major artifacts (impairing a diagnostic evaluation and necessitating a repeat scan with raised arms).

\section{Statistical analysis}

To compare time intervals between the two groups a twotailed $P$ value was calculated using the unpaired $t$ test. In case of significantly differing standard deviations, the unpaired $t$ test with Welch correction was applied. In addition, 95\% confidence intervals (CI) were calculated. The incidences of artifacts in the two groups were compared using the Mann-Whitney $U$ test. The age and sex distribution of patients in both groups was calculated and tested for degrees of difference using the unpaired $t$ test and Fisher's exact test, respectively. A $P$ value of less than 0.05 was considered significant.

\section{Results}

Patients

The mean age of all patients was 46.4 years (range 1689 years), the sex distribution was 65 men and 18 women. One protocol deviation occurred: a patient who was originally assigned to group 2 had multiple fractures of the arms that were clinically diagnosed before the CT scan. This patient's arms were not raised for the CT examination and this patient was subsequently assigned to group 1 . The mean age of the 39 patients in group 1 was 48.5 years, of the 44 patients in group 2, 44.4 years, a not-statisticallysignificant difference $(P=0.4)$. The sex distribution in group 1 was 29 men and 10 women, in group 2, 36 men and 8 women, also not a statistically significant difference $(P=0.44)$.

Time intervals

The results of time interval measurements are summarized in Table 3 . The mean scan time was $21 \mathrm{~min}$ in group 1 and $25 \mathrm{~min}$ in group 2. This difference was found to be statistically significant $(P=0.01)$. In group 1 , a repeat scan with raised arms was performed in $7 \%(3 / 44)$ of patients

Table 3 Scan time and repositioning time in minutes for CT scanning of multiple-trauma patients without (group 1) and with (group 2) repositioning

\begin{tabular}{llll}
\hline & Group 1 (no repositioning) & Group 2 (repositioning) & $P$ value and 95\% confidence interval \\
\hline Scan time & & & \\
Mean time & 21 & 25 & $P=0.01$ \\
Range & $12-37$ & $13-49$ & CI=1.02 to 7.58 \\
SD & 7 & 8 & \\
Repositioning time & & 8 & \\
Mean time & - & $3-20$ & \\
Range & & 3 & \\
SD & & & \\
\hline
\end{tabular}

CI-95\% confidence interval

${ }^{a}$ In group 1, a repeat scan with raised arms was performed in $7 \%(3 / 44)$ of patients due to major artifacts, which increased the mean scan time to $33 \min (P=0.47)$ 
due to major artifacts, increasing the mean scan time to $33 \mathrm{~min}$, which did not differ significantly from the mean scan time of the other patients in group $1(P=0.47,95 \%$ $\mathrm{CI}=-13.9$ to 25.08$)$ or of patients in group $2(P=0.23,95 \%$ $\mathrm{CI}=-9.45$ to 29.22 ). In group 2 , the mean repositioning time was $8 \mathrm{~min}$.

\section{Artifacts}

Artifacts due to arms and installations (Fig. 1) were only found in the upper abdomen, affecting evaluation of the liver and spleen. Significantly, more scans showed artifacts in group $1(62 \% ; 27 / 44)$ than in group $2(15 \% ; 6 / 39)$ $(P=0.0001)$ (Fig. 2). In group $1,38 \%$ (17/44) of CT scans exhibited no artifacts, $52 \%(23 / 44)$ had moderate artifacts (Fig. 3), and 10\% (4/44) had major artifacts (Fig. 4). A repeat scan with raised arms was performed in $7 \%(3 / 44)$ of patients due to major artifacts that did not allow conclusive evaluation of liver and spleen. In one case the arms could not be raised due to multiple fractures of the humerus and scapula. In group 2, 85\% (33/39) of CT scans showed no artifacts, $15 \%(6 / 39)$ showed moderate artifacts and no CT scan had major artifacts. No repeat scans were performed in group 2.

\section{Discussion}

In patients undergoing the novel $\mathrm{CT}$ protocol with repositioning from head-first to feet-first fewer artifacts in scans of the upper abdomen were found than in patients who were not. Patients who were repositioned also had a longer scan time than those undergoing the head-first protocol without repositioning.

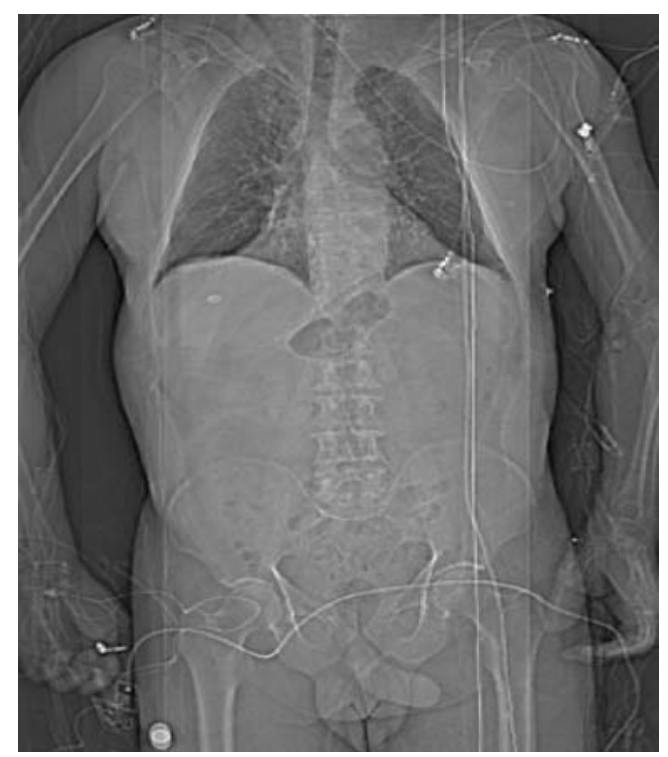

Fig. 1 Fifty-eight-year-old man after motor vehicle accident. CT scout image for body scan in head-first position with arms at the side. Arms, electrodes, and cables are within the scan field
Opinions in the literature differ regarding the proper positioning of multiple-trauma patients for CT scans. It is recommended in particular that arms be positioned above the head for a body scan to minimize artifacts, mainly in the upper abdomen [12]. Arms may also be crossed on the upper abdomen to reduce artifacts. An exception should be made for patients with clinically detectable fractures of shoulders and arms, because raising the arms in patients with fractures and/or dislocation of shoulders and/or arms may cause additional unnecessary pain, discomfort, or further progression of fractures and dislocations [14]. In this study, one patient had multiple fractures of the arms that were clinically diagnosed before the CT scan. This patient's arms were not raised during the CT examination so as not to engender harm to this patient who could have his injuries made worse by manipulating the upper extremities in repositioning and turning. Therefore, use of this technique with patient repositioning as described in this study is only recommended for patients without clinically suspected fractures and/or dislocation of shoulders and/or arms, which must be ruled out clinically before the CT scan. In this case, arms can be placed on foam sponge ramps by the patient's side at an elevation of approximately $30^{\circ}$ from the table [15]. This maneuver can even be performed using different inclination angles for each arm to minimize streak artifacts caused by arm bones being in direct opposition. The authors of another study did not reposition patients' arms to perform a whole-body single-pass trauma protocol [8]. This resulted in a reduced total radiation dose due to a reduction in redundant imaging at overlap zones between body segments, which occurs with segmental protocols. This did not, however, resolve the issue of artifacts.

Some centers use a rigid head-first CT protocol without patient repositioning for multiple-trauma patients, primarily to shorten scan times and, thus, to expedite emergency care $[12,14]$. Not surprisingly, in this position, arms and installations may cause beam hardening artifacts for the upper abdomen, thus concealing potentially life-threatening injury to the liver and spleen [16]. In the present study, artifacts occurred in more than half of patients without repositioning. In $10 \%$ of patients, the artifacts were major, meaning they did not allow diagnostic evaluation of the upper abdomen. In $7 \%$ of patients, an abdominal scan with raised arms had to be repeated, as determined on a case-tocase basis by the radiologist. Repeat scanning resulted in an increase of the scan time, mainly due to arm repositioning for relocation of infusion lines behind the gantry without rotating the patient. In anesthetized patients, the tracheal tube connections also had to be relocated behind the gantry. Furthermore, an additional bolus of contrast medium had to be administered.

In this study, a novel protocol was used in an attempt to reduce artifacts to a minimum. After scanning of the head and neck, the patient was rotated to a feet-first position on a plastic board below the vacuum cushion and arms were raised for the body scan. Optimization of multiple-trauma scanning protocols is thought to entail a trade-off between scan time and artifacts. It is not surprising, therefore, that 
Fig. 2 Distribution of artifacts in group 1 without repositioning compared to group 2 with repositioning. In group 1, artifacts occurred in $62 \%$ of scans, which was significantly more than in only $15 \%$ of scans in group 2 . *In $7 \%$ of patients in group 1 , a repeat scan had to be performed due to major artifacts

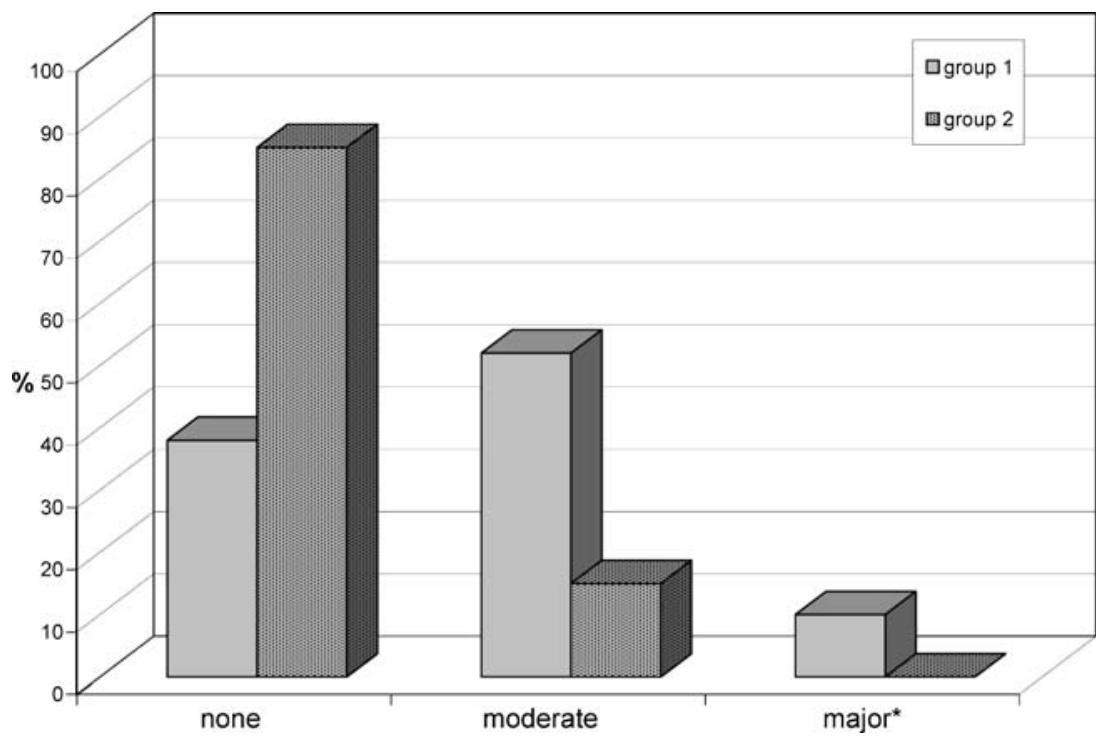

our repositioning for minimization of artifacts resulted in a prolongation of scan time. In our view, the mere 4-min scan-time difference between the two protocols used in the present study may not be of great relevance, as artifacts were significantly reduced.

As the time evaluation of this study shows, the mean scan times were $21 \mathrm{~min}$ without and $25 \mathrm{~min}$ with repositioning. However, the pure acquisition time for head/neck and body scan did not exceed $80 \mathrm{~s}$ for either protocol. The total scan time interval encompasses the planning of the examination based on the scout images, patient repositioning, application of contrast agent, and the radiologist's decision process to conclude the examination. Each of these elements may prolong the scan time. Because the times for planning of the examination and application of contrast agent did not differ between the two protocols, the difference in scan time was due not only to the repositioning time but also to the time the radiologist needed to decide to conclude the examination.

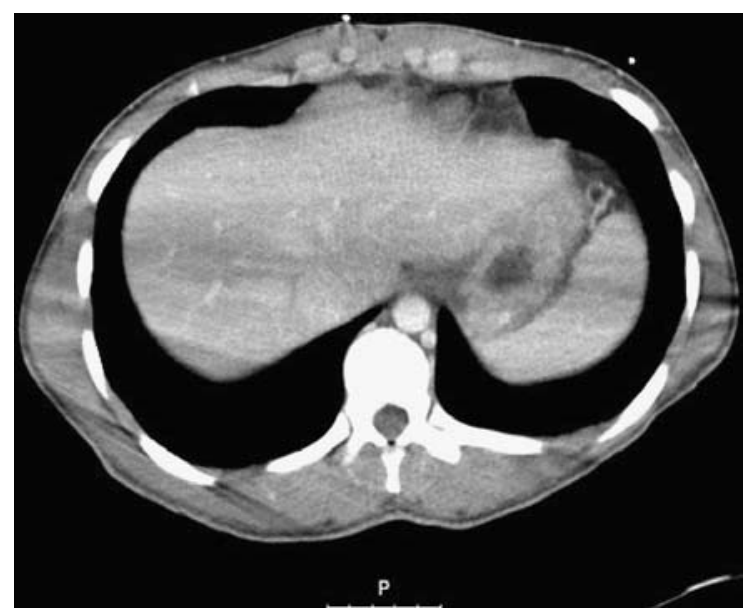

Fig. 3 Eighteen-year-old male patient involved in motor cycle accident. The body scan was performed in head-first position with arms at the sides, causing moderate streak artifacts within the upper abdomen as shown in this CT image
The repositioning procedure prolonged the scan time, but the procedure itself was simplified by keeping the multiple-trauma patients on a vacuum cushion, making it relatively easy to move them to the scanner table. The emergency personnel were instructed in advance when and how to rotate the patient on the $\mathrm{CT}$ table. The repositioning protocol also allowed the anesthesiologist to work without extensions for tubes and cables, because there was no need to pass installations through the gantry. In the present study, only moderate artifacts were observed in a small number of patients with repositioning. In contrast, major artifacts were observed using the standard protocol. This demonstrates that repositioning was able to minimize beam hardening artifacts from arms and installations and did not necessitate repeat scanning.

The term RUSH-CT applies to a protocol comprised of one pilot scan and three spiral scans. With this protocol, Linsenmaier et al. were able to shorten the scan time from 35 to $16 \mathrm{~min}$, allowing completion of $\mathrm{CT}$ within the first

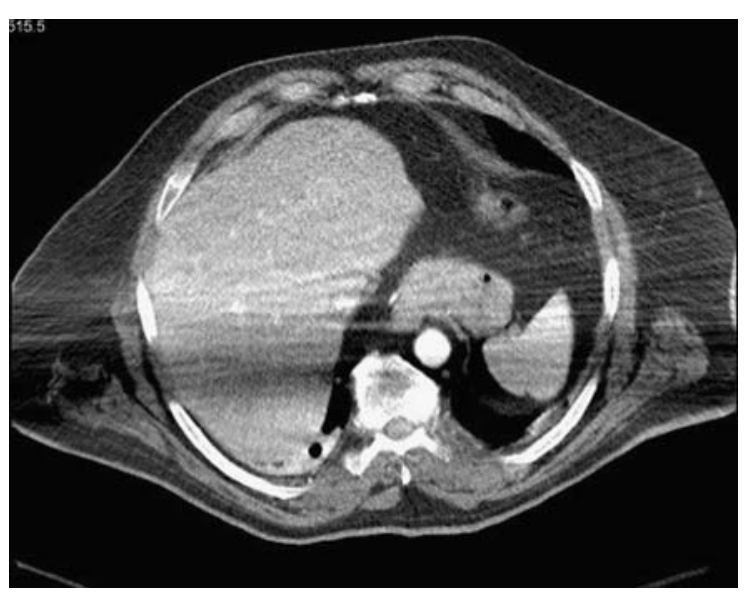

Fig. 4 Fifty-two-year-old male patient after motor vehicle accident CT image of the upper abdomen shows major beam hardening artifacts due to arms at the side of the body, thus concealing potentially life threatening injury, in this case, especially to the liver 
30 min after patient admission and thus facilitating early therapeutic intervention. Artifacts, however, compromised the image quality [11]. Another work group applied a single-pass whole-body protocol with multi-row detector CT that shortened room time from the 65 min needed to single detector $\mathrm{CT}$ to $23 \mathrm{~min}$ without grading artifacts [15].

Overall, there exist several different scanning protocols for multiple-trauma patients concerning patient positioning such as whole body scan with elevated arms or repositioning of the arms, e.g., ventrally on the chest or through the gantry after the head-and-neck scan without rotation of the patient $[12,14]$. However, the present results and those of others show that no consensus yet exists regarding the optimal protocol for CT scans of multiple-trauma patients. For modern multi-row detector $\mathrm{CT}$, the pure acquisition time can be disregarded and the complex chain of processes during the room and diagnostic time needs to be further elucidated. The time discrepancies between the different scanning protocols underscore the need for further optimization of workflow, including the movement and repositioning of multiple-trauma patients. Emergency personnel have to be aware that teamwork and efficiency are essential contributors to patient outcome. It must be noted, though, that study results are, to a certain extent, center-specific due to differences in workflow and definitions of time intervals.

In conclusion, this novel multiple-trauma CT scanning protocol with patient repositioning achieves a higher image quality with significantly fewer artifacts than without repositioning. However, the scan time is slightly increased with patient repositioning, compared to the protocol without repositioning.

\section{References}

1. Novelline RA, Rhea JT, Rao PM, Stuk JL (1999) Helical CT in emergency radiology. Radiology 213:321-339

2. Jelly LM, Evans DR, Easty MJ, Coats TJ, Chan O (2000) Radiography versus spiral CT in the evaluation of cervicothoracic junction injuries in polytrauma patients who have undergone intubation. Radiographics 20(Spec No):S251S259; Discussion S260-S262
3. Daffner RH (2001) Helical CT of the cervical spine for trauma patients: a time study. AJR Am J Roentgenol 177:677-679

4. Harris JH (2001) Reflections: emergency radiology. Radiology 218:309-316

5. Mahesh M (2002) Search for isotropic resolution in CT from conventional through multiple-row detector. Radiographics 22:949-962

6. Okamoto K, Norio H, Kaneko N, Sakamoto T, Kaji T, Okada Y (2002) Use of early-phase dynamic spiral computed tomography for the primary screening of multiple trauma. Am J Emerg Med 20:528-534

7. Poletti PA, Wintermark M, Schnyder P, Becker CD (2002) Traumatic injuries: role of imaging in the management of the polytrauma victim (conservative expectation). Eur Radiol 12:969-978

8. Ptak T, Rhea JT, Novelline RA (2003) Radiation dose is reduced with a single-pass whole-body multi-detector row CT trauma protocol compared with a conventional segmented method: initial experience. Radiology 229:902-905

9. Nunez DB, Ledbetter MS, Farrell L (2002) Dedicated CT scanner in an emergeny department: quantification of factors that contribute to lack of use. AJR Am J Roentgenol 179: $859-862$

10. Ruchholtz S, Zintl B, Nast-Kolb D, Waydhas C, Lewan U, Kanz KG, Schwender D, Pfeifer KJ, Schweiberer L (1998) Improvement in the therapy of multiply injured patients by introduction of clinical management guidelines. Injury 29: $115-129$

11. Linsenmaier U, Rieger J, Brandl T, Niethammer M, Scherf C, Rock C, Pfeifer KJ (2000) New method for fast spiral CT of trauma patients-RUSH CT. Emerg Radiol 7:135-141

12. Leidner B, Adiels M, Aspelin P, Gullstrand P, Wallen S (1998) Standardized CT examination of the multitraumatized patient. Eur Radiol 8:1630-1638

13. Gralla J, Spycher F, Pignolet C, Ozdoba C, Vock P, Hoppe H (2005) Evaluation of a 16-MDCT scanner in an emergency department: initial clinical experience and workflow analysis. AJR Am J Roentgenol 185:232-238

14. Linsenmaier U, Krotz M, Hauser H, Rock C, Rieger J, Bohndorf K, Pfeifer KJ, Reiser M (2002) Whole-body computed tomography in polytrauma: techniques and management. Eur Radiol 12:1728-1740

15. Ptak T, Rhea JT, Novelline RA (2001) Experience with a continuous, single pass whole-body multidetector CT protocol for trauma: the three-minute multiple trauma scan. Emerg Radiol 8:250-256

16. Matthes G, Stengel D, Seifert J, Rademacher G, Mutze S, Ekkernkamp A (2003) Blunt liver injuries in polytrauma: results from a cohort study with the regular use of whole-body helical computed tomography. World J Surg 27:1124-1130 